\title{
Séquence de dépôts binaire et discontinuités associées
}

\section{Exemple des séries crétacé-tertiaire du bassin Maghrébin et de l'Atlas saharien, Algérie du Nord}

\author{
M.E. Baghli* et M.M. Mouhoubi \\ Sonatrach, Institut Algérien du Pétrole, Département Géologie, avenue du 1er Novembre, 35000 Boumerdès - Algérie \\ e-mail : khbaghli@yahoo.fr-mmouhoubi@yahoo.fr \\ *Auteur à contacter
}

Résumé - Séquence de dépôts binaire et discontinuités associées. Exemple des séries crétacétertiaire du bassin Maghrébin et de l'Atlas saharien, Algérie du Nord - Une séquence de dépôts binaire a été élaborée à partir d'observations de terrain. Elle s'applique à différentes échelles et à différents milieux de dépôts. La séquence binaire est composée de deux séquences. Chacune d'elles est subdivisée en deux cycles. Les cycles inférieurs représentent les $3 / 5^{\mathrm{e}}$ en durée et les cycles supérieurs les $2 / 5^{\mathrm{e}}$. Au total, cela fait quatre cycles. Dans le détail, cette séquence binaire comprend dix termes de faciès délimités par dix discontinuités dont quatre sont importantes. Ces dernières délimitent les quatre cycles. On distingue deux «maximum flooding surface» (m.f.s.) au centre des deux cycles supérieurs.

\footnotetext{
Abstract - Binary Sequence of Deposits and Associated Discontinuities. Example of the Series Cretaceous-Tertiary of the Maghrebin Basin and of the Saharian Atlas, North Algeria - A binary sequence of deposits was elaborate starting from the observations of ground. It applies to various scales and various of deposits media. The binary sequence is made up of two sequences. Each sequence is subdivided in two cycles. The lower cycles account for the three fifths in duration and the higher cycles the remaining two fifths. On the whole, that made four cycles. In detail, this binary sequence includes ten terms of facies delimited by ten discontinuities of which four among they are significant. These last delimit the four cycles. One distinguishes two m.f.s. or maximum flooding surface in the center from the two higher cycles.
} 


\section{INTRODUCTION}

L'objectif de ce travail est de contribuer à établir un modèle de séquence de dépôts à différentes échelles, dans le temps et dans l'espace, à partir d'observations de terrain. Il s'agit, d'une part, de délimiter les séquences, déterminer leurs différents ordres et de hiérarchiser les discontinuités; d'autre part, comprendre leur signification géodynamique et accéder au temps. Nous nous proposons de trouver une explication et une logique entre les séquences et les discontinuités, comme la variation du niveau de la mer, la tectonique, les fluctuations du substratum ou la source d'apport.

Nous avons travaillé dans le bassin Maghrébin et l'Atlas saharien qui présentent les différents ordres de séquences. L'exemple des dépôts de plaine sous-marine du Crétacé supérieur à l'Oligocène du Djebel Zima à $30 \mathrm{~km}$ au sud-est d'Alger, d'Azeffoun et de Médjana, donne une bonne illustration.

Afin de voir l'évolution latérale des séquences à l'échelle du bassin, d'autres séries sur les marges du bassin ont été étudiées: la marge eurasienne au Nord dans la région d'Ighil Oudlès et la marge africaine au Sud dans les régions de Bou-Saada et d'Aflou.

\section{LES SÉQUENCES ÉTUDIÉES SUR LE TERRAIN}

À partir de levés de coupes au $1 / 100^{\mathrm{e}}$ et au $1 / 40^{\mathrm{e}}$ effectués sur le terrain, nous proposons un modèle de séquence de dépôts à cinq termes $a, b, c, d$ et e. Les faciès de ces séries sédimentaires du Djebel Zima, d'Azeffoun et de Medjana s'étalent du Campanien-Maestrichtien à l'Oligocène. Ce sont des dépôts de la plaine sous-marine.

Deux types de séquences ont été identifiés en comparant les faciès des différents termes: la séquence de type a à dominante terrigène de l'Oligocène, et la séquence de type $b$ à dominante carbonatée du Lutétien.

Les strates du Lutétien (fig. 1 et photo jointe) de la série du Djebel Zima ont particulièrement attiré notre attention. Ce sont des bancs carbonatés pluridécimétriques appelés doublets ou couplets. La partie inférieure de ces bancs est à dominante carbonatée et la partie supérieure, plus réduite, est à dominante terrigène.

C'est cette répartition, unique en son genre, de la base du banc carbonatée ( $3 / 5^{\mathrm{e}}$ en épaisseur) et du sommet terrigène ( $2 / 5^{\mathrm{e}}$ en épaisseur), qui nous a permis de déduire une logique valable également dans les séquences des autres étages étudiés. Ceci justifie le choix de la coupe du Lutétien par rapport à d'autres qui ne présentent pas cette succession proposée dans cette note.

\subsection{La séquence de type a}

La séquence de type a (fig. 2), décimétrique à décamétrique, est composée de deux cycles. Le cycle inférieur est constitué de trois termes $a, b$ et $c$. Un terme central terrigène $b$ de grès arkosiques dits faciès gréso-micacés, est encadré par deux termes carbonatés a et c.

Le cycle supérieur est constitué des termes $d$ et e. Ce cycle comprend une strate ou bande terrigène $\mathrm{d}$, de grès-quartzites dits faciès numidiens, surmonté d'une strate ou bande carbonatée e. Le second cas observé dans ce type de séquence consiste en un banc de grès-quartzites $\mathrm{d} 2$ encadré par des calcilutites ou des marnes $\mathrm{d} 1 \mathrm{et} \mathrm{e}$.

Les épaisseurs des cycles inférieur et supérieur sont respectivement de $3 / 5^{\mathrm{e}}$ et de $2 / 5^{\mathrm{e}}$.

Des pélites rouges ou noires du bruit de fond ponctuent les passages entres les grès et les carbonates ou entre les termes $a, b, c, d$ et e.

On distingue 5 discontinuités principales $\varphi_{1}, \varphi_{2}, \varphi_{3}, \varphi_{4}$ et $\varphi_{5}$ entre les cinq termes $\mathrm{a}, \mathrm{b}, \mathrm{c}, \mathrm{d}$ et e. Plus précisément au sommet des pélites rouges ou noires qui délimitent les termes entre eux.

Le troisième cas de séquence de type a est le plus compliqué à décrire. Mais il est intéressant pour la suite des travaux. Il montre la même répartition en cinq termes $\mathrm{a}, \mathrm{b}, \mathrm{c}, \mathrm{d}$ et e. La nouveauté consiste en l'observation dans chacun de ces termes d'ordre 1 , cinq sous-termes a, b, c, d et e d'ordre 2 emboîtés dans chacun des termes a, b, c, d et e d'ordre 1 (fig. 2).

On dénombre cinq termes comprenant chacun cinq soustermes. Au total, cela fait vingt cinq strates ou bandes de faciès terrigènes, carbonatés et pélitiques regroupés en cinq termes a, b, c, d et e d'ordre 1 .

Cet emboîtement n'est pas aisé à observer sur le terrain.

Pour les trois types de séquences, les cycles inférieurs sont à faciès oxygénés et composés de pélites claires, vertes et rouges, du bruit de fond. Les pélites rouges sont généralement bioturbées.

Les cycles supérieurs montrent au contraire des pélites sombres ou noires, riches en matière organique, et une diminution du pourcentage de carbonates dans le terme d. Ce sont des faciès confinés avec une diminution dans le pourcentage d'oxygène et une absence de bioturbation.

\subsection{La séquence de type $b$}

La séquence de type b (fig. 2) est celle du Lutétien. Elle est rarement décrite dans la littérature. C'est aussi une séquence pluridécimétrique composée de cinq termes a, b, c, d et e. La différence avec la séquence de type a réside dans le terme $\mathrm{b}$ qui est carbonaté comme les termes a et c. C'est une séquence qui présente une partie inférieure carbonatée et une partie supérieure terrigène. Cette partie inférieure se compose de trois termes carbonatés $\mathrm{a}, \mathrm{b}$ et $\mathrm{c}$ séparés par des joints argileux. La partie supérieure, plus réduite, est constitué de deux termes: le terme inférieur d gréseux et le terme supérieur e carbonaté. Ce dernier terme n'est pas systématiquement présent dans la séquence de type $b$. 


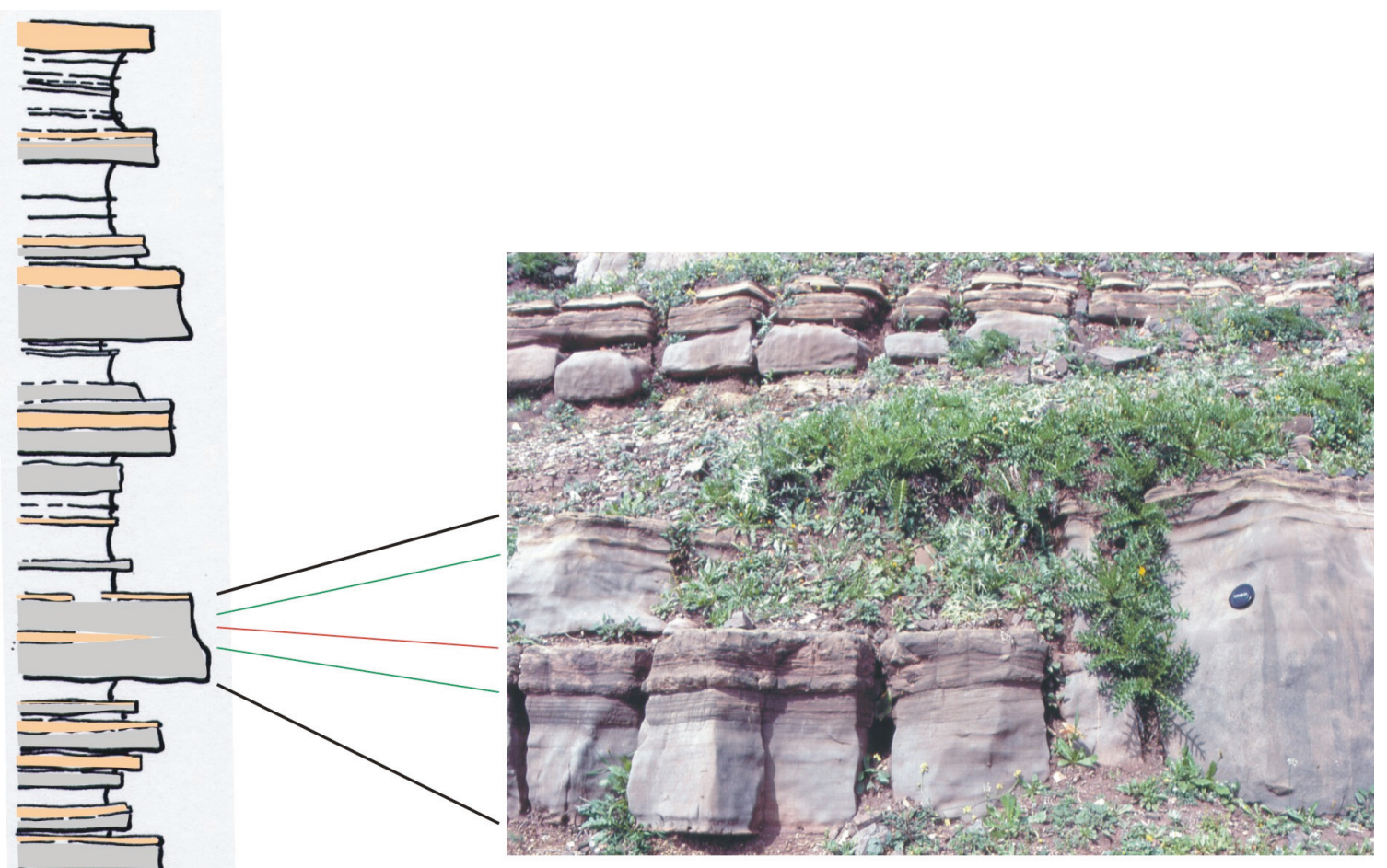

Le modèle de séquence de dépôts binaire, deux doublets ou deux couplets

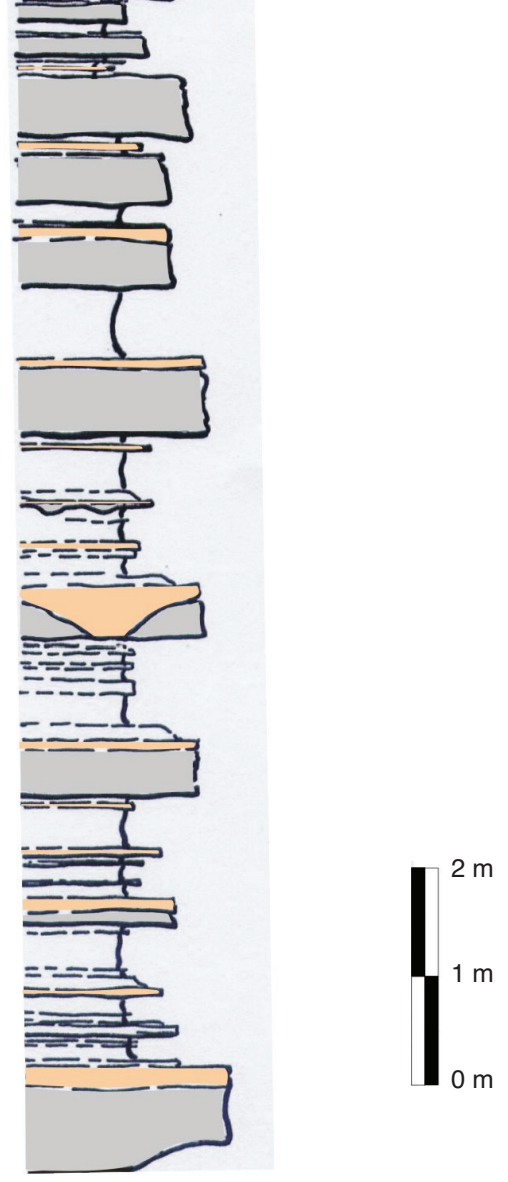

Détritique terrigène

Carbonates

Bruit de fond : pélites rouges

Figure 1

Le Lutétien de la série du Djebel Zima. The Lutetian of the Djebel Zima's serie. 
Le second cas de la séquence de type b se rapporte au terme d gréseux qui peut présenter une base carbonatée. On distingue un sous-terme $\mathrm{d} 1$ pour le faciès carbonaté et un sous-terme $\mathrm{d} 2$ pour le faciès terrigène. La base du cycle inférieur de cette séquence $b$ présente souvent des microbrèches. La base du cycle supérieur montre plutôt des convolutes. Cette disposition montre une similitude avec les termes de la séquence de Bouma (1962).

On note aussi comme pour la séquence de type a cinq discontinuités principales sédimentaires et/ou tectoniques $\varphi_{1}$, $\varphi_{2}, \varphi_{3}, \varphi_{4}$ et $\varphi_{5}$. Ces discontinuités apparaissent au sommet des pélites rouges ou noires délimitant les termes a, b, c, d et e.

\section{DISCUSSION}

\subsection{Les cycles connus dans la littérature}

Dans la littérature, Gilbert (1895) a étudié les alternances du Crétacé supérieur du Colorado et les a liées aux oscillations climatiques.
Les travaux de De Boer et Wonders (1984) rapportent à 20 ka la durée d'un cycle élémentaire calcaire-marne. Ils dénombrent ainsi dans le Cénomanien 332 doublets ou couplets calcaire-marne. On multiplie ce nombre de couplets par $20 \mathrm{ka}$. On obtient une durée de 6640000 ans. Soit l'ordre de grandeur de cet étage qui est de 4 à 6 millions d'années.

Les Pac's de Goodwin et Anderson (1985) sont des séquences de $100 \mathrm{ka}$. Ces cycles sont ceux de Milankovitch. On distingue la précession de $20 \mathrm{ka}$, l'obliquité de $40 \mathrm{ka}$, l'excentricité à court terme de 100 ka et l'excentricité à long terme de $400 \mathrm{ka}$. Ce dernier cycle n'a pu être vérifié sur le terrain.

Un autre cycle plus récent est rapporté par Rhode et Muller (2005) d'une durée de 62 Ma. Il est basé sur les pourcentages d'extinction des populations d'espèces dans l'échelle stratigraphique. Aucune origine n'est avancée pour ce cycle. Pour ce dernier, notre estimation empirique sur le terrain est de 62,5 Ma.

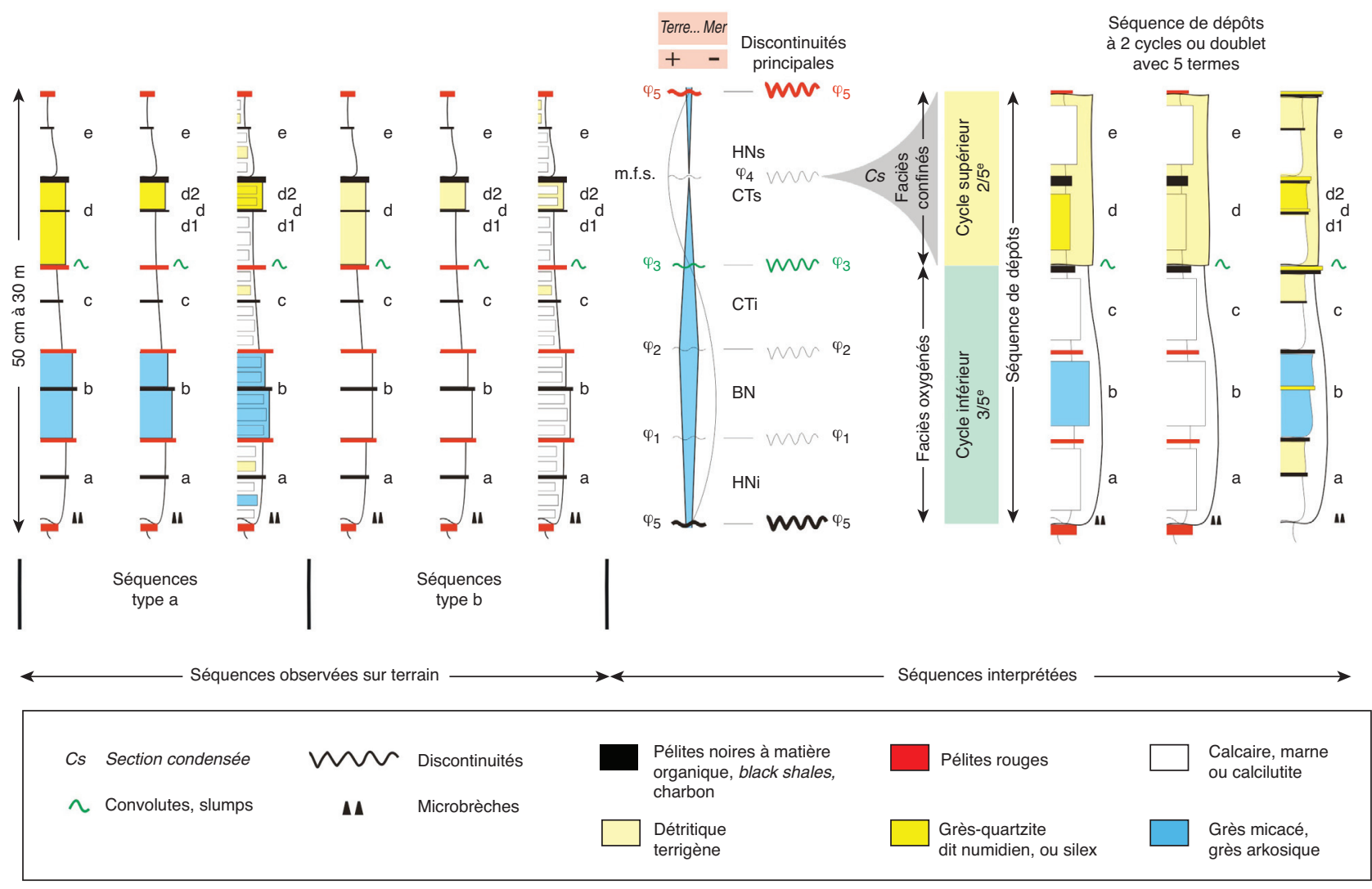

Figure 2

Les séquences observées sur terrain. Sequences observed on ground. 


\subsection{L'estimation de la durée des strates sur le terrain}

L'estimation de la durée des strates a été effectuée en divisant la durée d'un étage par le nombre total de strates de ce même étage. De la même manière, la durée d'un paquet de strates a été estimée en divisant la durée d'un étage par le nombre de paquets de strates. Il s'agit d'appliquer ce raisonnement uniquement à un étage qui a préservé toutes ses strates. Autrement dit, s'il manque une partie des strates pour une raison tectonique ou par condensation, cette estimation devient erronée (pour plus de détails, se référer à la figure 3 du chapitre 3.2).

La durée des strates a été estimée à $2 \mathrm{ka}, 10 \mathrm{ka}, 20 \mathrm{ka}$ ou 50 ka pour les formations épaisses, du Bartonien à l'Oligocène. Dans les formations condensées du Lutétien, les strates auraient une durée de $25 \mathrm{ka}, 50 \mathrm{ka}, 75 \mathrm{ka}, 125 \mathrm{ka}$ et $250 \mathrm{ka}$. Dans le Priabonien et l'Oligocène inférieur, les paquets pluridécimétriques à métriques de strates auraient une durée de $50 \mathrm{ka}$ et 100 ka. Ces derniers sont nommés dans la littérature des Pac's ou cyclothèmes. Nous émettons des réserves sur les valeurs des durées estimées. Il s'agit de propositions pour de nouvelles pistes de recherche que de solutions absolues.

\subsection{Les analyses effectuées sur les pélites noires}

Des analyses ont été effectuées et interprétées par Djarnia (1990, à notre demande). Il s'agit de pélites noires de l'Oligocène supérieur du Djebel Zima. Les résultats font ressortir que le rapport pristane/phytane est de 2,8, le pourcentage de carbone organique de 0,93 et le pourcentage de carbone minéral de 0,04 . Il s'agit d'une matière organique d'origine continentale, non dégradée (non oxydée).

\section{INTERPRETATION}

\subsection{Les séquences de dépôts binaires}

Les séquences de dépôts observées et décrites dans le premier chapitre, ne constituent pas à elles seules le modèle complet de séquence de dépôts. Elles doivent être associées à une autre séquence située en dessous ou en dessus, pour constituer une séquence de dépôts binaire. Autrement dit, il faut réunir deux séquences de type a (ou de type b) de mêmes durées (fig. 3) pour faire des séquences de dépôts binaires. De là est né le concept de modèle de séquence de dépôts binaire. Cela fait donc deux séquences, chacune d'elles étant subdivisée en deux cycles. Le cycle inférieur constitue les $3 / 5^{\mathrm{e}}$ en épaisseur et en durée. Le cycle supérieur constitue les $2 / 5^{\mathrm{e}}$ en épaisseur et en durée. Au total, il y a quatre cycles dans une séquence de dépôts binaire.

En prenant comme exemple la séquence de dépôts binaire $100 \mathrm{ka}$ (fig. 3), on distingue 10 termes (a, b, c, d et e puis a', b', c', d' et e'), 10 discontinuités $\left(\varphi_{1}, \varphi_{2}, \varphi_{3}, \varphi_{4}\right.$ et $\varphi_{5}$ puis $\varphi_{1}$, $\varphi_{2}, \varphi_{3}, \varphi_{4}$ et $\varphi_{5}$ ) et 4 cycles ( 2 cycles inférieurs et 2 cycles supérieurs).

En appliquant la terminologie de Vail et al. (1977) à ces séquences binaires (fig. 2 et 3), on déduit ce qui suit :

- les termes a, e et a', e' sont du haut niveau marin HN. Il faut préciser haut niveau inférieur HNi pour a et a' et haut niveau supérieur HNs pour e et e';

- les termes b et b' correspondent au bas niveau BN;

- les termes $\mathrm{c}, \mathrm{d}$ et c', d'appartiennent au cortège transgressif CT. Là aussi, il faut préciser cortège transgressif inférieur CTi pour c et c' et cortège transgressif supérieur CTs pour d et d'.

Les deux m.f.s. ou maximum flooding surface se situent au centre des deux cycles supérieurs. Elles sont synchrones aux discontinuités $\varphi_{4}$.

C'est dans les cycles supérieurs des séquences binaires que se développent les pélites noires riches en matière organique et les black-shales. À titre d'exemple, les grès-quartzites dits numidiens et leur bruit de fond à pélites noires riches en matières organiques se manifestent dans les termes $\mathrm{d}$ et d' des cycles supérieurs des séquences.

Ces pélites noires riches en matière organique de l'Oligocène sont cycliques et non aléatoires. Cette préservation de la matière organique s'établit tous les $10 \mathrm{ka}$ dans une séquence de $100 \mathrm{ka}$ quand les conditions sont réunies.

Ce cycle supérieur est celui de la section condensée ou «condensed section» qui est synchrone à la transgression, à la m.f.s. et à la discontinuité $\varphi_{4}$.

\subsection{L'estimation des différents ordres de séquences de dépôts binaires}

L'estimation des différents ordres de séquences de dépôts binaires et emboîtées (fig. 3) a été déduite empiriquement. C'était l'un des buts de ce travail de hiérarchiser ces différents ordres. Il fallait déduire que les durées des différentes séquences rapportées dans la littérature sont des multiples de cinq. Auparavant, il fallait comprendre que la séquence (quel que soit son ordre) n'est pas isolée mais toujours associée à une seconde séquence, en dessous ou en dessus.

De la figure 3, nous pouvons déduire qu'il faut cinq séquences binaires d'un ordre quelconque pour faire une séquence binaire de l'ordre supérieur. Par exemple, il faut cinq séquences de $62,5 \mathrm{Ma}$ de $2^{\mathrm{e}}$ ordre pour faire une séquence binaire de $312,5 \mathrm{Ma}$ de $1^{\mathrm{er}}$ ordre.

Sept ordres de séquences de dépôts binaires et emboîtées ont été déterminés. Du plus grand au plus petit, on distingue les séquences suivantes :

- $1^{\mathrm{er}}$ ordre de 312,5 Ma entre deux pangées,

- $2^{\mathrm{e}}$ ordre de 62,5 Ma ou durée d'un système,

- $3^{\mathrm{e}}$ ordre de 12,5 Ma ou étage,

- $4^{\mathrm{e}}$ ordre de 2,5 Ma,

- $5^{\mathrm{e}}$ ordre de $500 \mathrm{ka}$, 


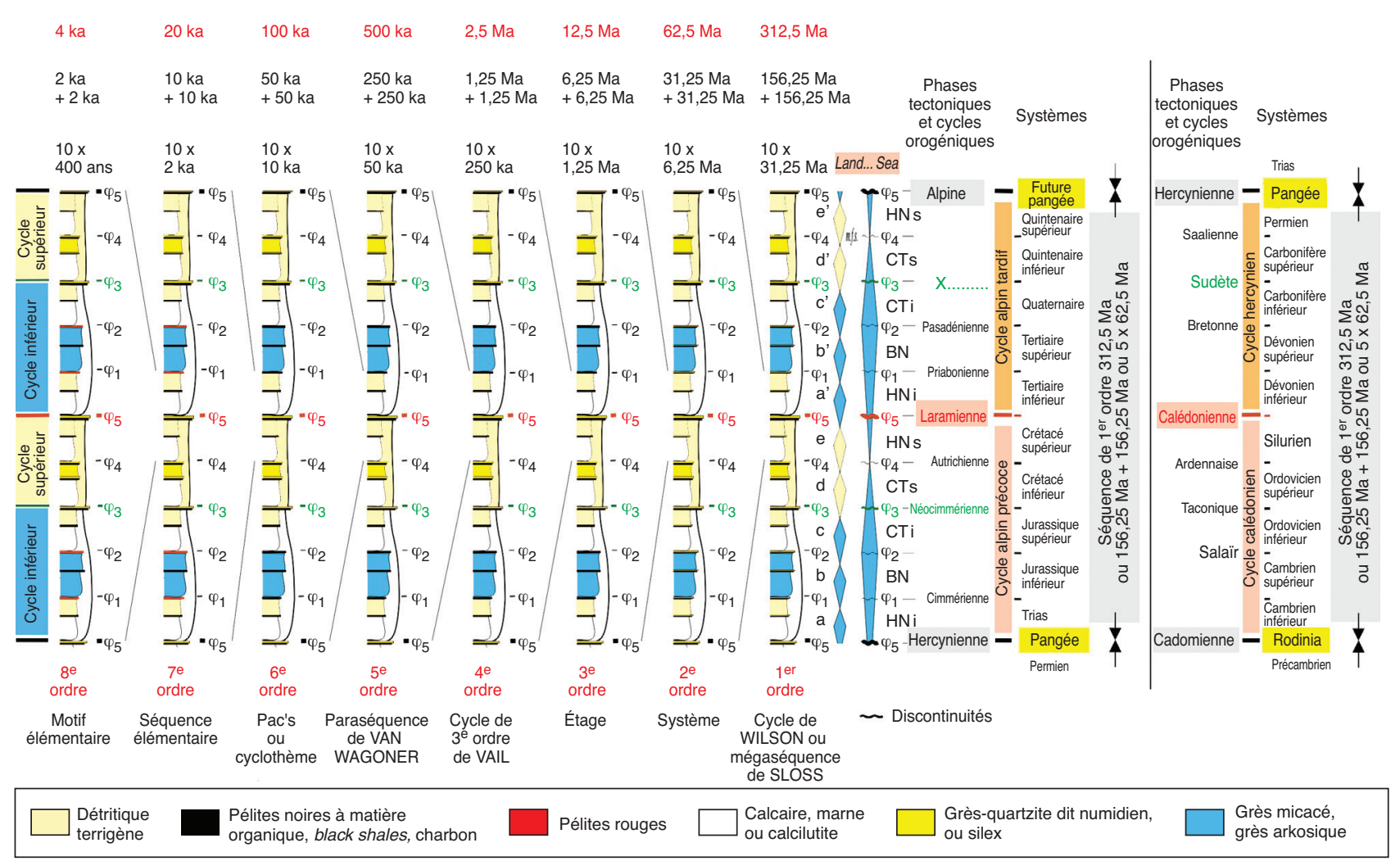

Figure 3

Les différents ordres de séquences binaires emboîtées.

Various orders of encased binary sequences.

- $6^{\mathrm{e}}$ ordre de $100 \mathrm{ka}$,

- $7^{\mathrm{e}}$ ordre de $20 \mathrm{ka}$ ou séquence élémentaire et

- 8 e ordre de 4 ka ou motif élémentaire.

L'équivalent du $1^{\mathrm{er}}$ ordre correspondrait au cycle de Wilson, de l'ouverture à la fermeture des plaques, entre deux pangées. Il peut correspondre aussi à la mégaséquence de Sloss (1963, 1988).

Pour Sloss, le $2^{\mathrm{e}}$ ordre de séquence correspond à la moitié de la mégaséquence. Dans cette note, le second ordre correspond au $1 / 5^{\mathrm{e}}$ de la séquence de $1^{\mathrm{er}}$ ordre de $312,5 \mathrm{Ma}$, soit 62,5 Ma (fig. 3).

Cette durée a aussi été signalée dans les travaux de Rohde et Muller (2005) de l'Université de Berkelet. Pour ces derniers, ce cycle de 62 Ma est basé sur les pourcentages d'extinctions d'espèces pour toute l'échelle stratigraphique. Aucune origine pour ce cycle n'est signalée par ces auteurs. Nous nous posons tout de même une question. Pourquoi deux séquences (l'une inférieure, l'autre supérieure) pour former une séquence de dépôts binaire? Cette binarité s'observe à toutes les échelles.

En conclusion de ce chapitre, nous pouvons dire que ce que l'on observe à grande échelle se retrouve aussi à petite échelle. Nous pensons que ces séquences, quelque soit leur ordre, s'inscrivent bien dans un modèle séquentiel unique. Il s'agit de nouvelles pistes de recherche et non des valeurs de durées absolues et indiscutables. Le débat reste ouvert.

\subsection{L'échelle stratigraphique séquentielle et la hiérarchie des différents ordres de discontinuités}

Une échelle stratigraphique séquentielle (fig. 4) basée sur le modèle de séquence de dépôts binaire a été élaborée. Nous avons hiérarchisé les différents ordres de discontinuités à l'échelle des séquences et mégaséquences de dépôts.

À l'échelle des plaques, pour le $1^{\text {er }}$ ordre de $312,5 \mathrm{Ma}$, on retrouve les dix discontinuités ou phases tectoniques dont quatre sont importantes et délimitent quatre cycles. Les m.f.s se situent aux centres des cycles supérieurs et sont synchrones des discontinuités $\varphi_{4}$. Les dix discontinuités ont été hiérarchisées dans une fourchette allant de un à quatre. Il existe encore des ordres inférieurs (5 et 6) pour les étages et sous-étages. Nous ne les avons pas reportés par manque de place sur les figures. 


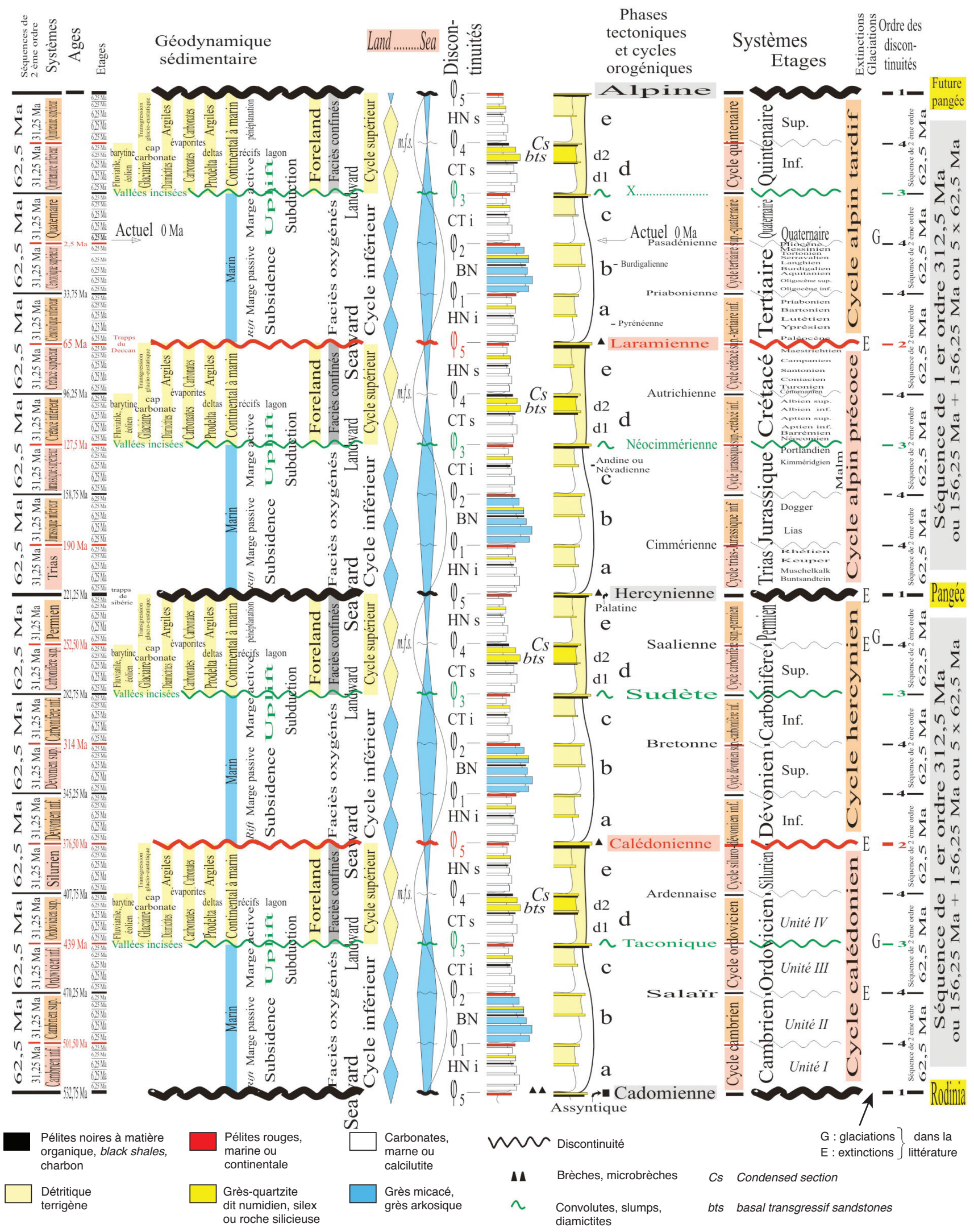

Figure 4

Échelle stratigraphique séquentielle.

Sequential stratigraphic scale. 
C'est aussi le modèle de Sloss (1988) avec quatre cycles et quatre discontinuités (unconformities) ou phases tectoniques. On arrive pratiquement aux mêmes résultats, pour les quatre discontinuités importantes, par des approches différentes.

Nous pensons que ce serait un modèle universel où toutes les séquences, décrites dans la littérature, s'intègreraient dans une séquence de dépôts binaire avec dix discontinuités dont quatre sont importantes. C'est ce que recherchent les spécialistes de la stratigraphie séquentielle.

\subsubsection{La mégaséquence de $1^{\mathrm{er}}$ ordre de $312,5 \mathrm{Ma}$ de l'ère primaire}

La mégaséquence de dépôts binaire de 312,5 Ma est appliquée ici à toute l'ère primaire (fig. 4). Les limites de cette mégaséquence sont d'ordre 1 (en noir) et correspondent aux phases assyntique et palatine, ou encore aux orogènes rodinia et pangée. Cette mégaséquence binaire est composée de deux séquences de même durée, soit 156,25 Ma chacune. La séquence calédonienne et la séquence hercynienne sont délimitées par la phase calédonienne d'ordre 2 (en rouge).

Ces deux séquences se subdivisent à leur tour en deux cycles. Les cycles inférieurs représentent les $3 / 5^{\mathrm{e}}$ en durée, soit 93,75 Ma. Les cycles supérieurs ont une durée de $2 / 5^{\mathrm{e}}$, soit 62,5 Ma.

C'est à la limite des cycles inférieurs et supérieurs des deux séquences qu'on retrouve les phases taconique et sudète $\varphi_{3}$ d'ordre 3 (en vert). C'est le même schéma que pour les séquences métriques, de $100 \mathrm{ka}$ de $6^{\mathrm{e}}$ ordre (fig. 2, 3), où l'on observe des convolutes ou des slumps dans la discontinuité $\varphi_{3}$, toute proportion gardée. Cette observation est importante dans la géodynamique sédimentaire.

Dans le détail, on retrouve les dix termes a, b, c, d, e et a', b', c', d', e' pour les deux séquences, séparés par dix discontinuités ou phases tectoniques d'ordre 4 (en noir). On peut citer, par exemple, les phases salaïr, ardennaise, bretonne et saalienne.

Les dix phases tectoniques ne se produisent pas d'une manière aléatoire dans le temps. Il s'agit d'une succession de phases répétitives tous les 31,25 Ma et 62,5 Ma. Le second battement est plus intense et délimite un cycle de 62,5 Ma.

Le cycle de 31,25 Ma se répète dix fois pour faire un mégacycle complet de 312,5 Ma. Le cycle de 62,5 Ma se répète cinq fois pour faire un cycle de 312,5 Ma. Les durées de 31,25 Ma correspondent à celles du Silurien et du Permien. Les durées de 62,5 Ma sont celles des systèmes cambrien, ordovicien, dévonien et carbonifère.

\subsubsection{La mégaséquence de $1^{\mathrm{er}}$ ordre de $312,5 \mathrm{Ma}$ à partir du Trias}

Le raisonnement ci-dessus vaut pour l'autre cycle de Wilson qui regroupe les ères secondaire, tertiaire et quaternaire (fig. 4). La figure 4 montre aussi un cycle de $1^{\text {er }}$ ordre de 312,5 Ma depuis le Trias. Les limites de cette mégaséquence sont d'ordre 1 (en noir) et correspondent aux phases palatine et alpine, ou encore aux orogènes de la pangée et de la "future pangée".

Cette mégaséquence est composée de deux séquences de même durée, soit 156,25 Ma chacune. La séquence alpine précoce et la séquence alpine tardive sont délimitées par la phase laramienne d'ordre 2 (en rouge). Ces deux séquences se subdivisent à leur tour en deux cycles. Les cycles inférieurs représentent les $3 / 5^{\mathrm{e}}$ en durée, soit $93,75 \mathrm{Ma}$. Les cycles supérieurs ont une durée de $2 / 5^{\mathrm{e}}$, soit $62,5 \mathrm{Ma}$.

C'est à la limite des cycles inférieurs et supérieurs des deux séquences, qu'on retrouve les phases Néocimmérienne et X (ainsi nommée pour la circonstance) d'ordre 3 (en vert).

Dans le détail, on retrouve les dix termes a, b, c, d, e et a', b', c', d', e' pour les deux séquences, séparés par dix discontinuités ou phases tectoniques d'ordre 4 (en noir). On peut citer par exemple les phases cimmérienne, autrichienne, priabonienne et pasadénienne.

\subsection{L'architecture d'une mégaséquence de dépôts binaire}

La géométrie de la mégaséquence de dépôts binaire, avec l'architecture des corps sédimentaires (fig. 5), met en évidence deux phénomènes.

Sur le plan géodynamique, à l'échelle des plaques, on penserait à un basculement dans le temps du substratum tantôt vers l'océan, tantôt vers le continent. Les variations de la pente du substratum seraient aussi importantes que les variations du niveau relatif de la mer. Ce basculement serait en phase avec des périodes de subsidence (termes a, b et a', b') et des périodes de remontée du substratum ou uplift (termes c, d et c' d').

Les périodes de subsidence s'expriment par des séries sédimentaires stables et épaisses, et des faciès oxygénés.

Les périodes de remontée du substratum montrent des séries condensées ou condensed section et des faciès confinés, ainsi qu'une instabilité du substratum qui s'exprime par une tectonique synsédimentaire avec des slumps et des convolutes.

Les termes e et e' des cycles supérieurs sont aussi les périodes de transgression comme le Silurien et le Crétacé supérieur avec un développement de la matière organique ou black-shales. Les m.f.s. se situent au centre de ces cycles supérieurs et sont synchrones des discontinuités $\varphi_{4}$.

Sur la figure 5, le continent (land) est représenté à gauche. Les termes e, a' et e', a sont en downlap et les termes c, d et c', d' en onlap.

En suivant le raisonnement de Cross (1988), les termes a, b et a', b' des cycles inférieurs tendent vers l'océan ou seaward stepping. Les termes c, d, et c', d' tendent vers le continent ou landward stepping. 


\section{4 ÉLARGISSEMENT DU DÉBAT}

\subsection{Le problème des grès numidiens dans le bassin Maghrébin}

Les directions des paléocourants, relevés à la base des bancs gréso-micacés de l'Oligocène, sont de Nord $125^{\circ}$.

Les grès-quartzites matures ou faciès dits numidiens ne montrent pas de figures de courant pour mesurer la direction de leur source d'apport. Cette dernière, du Nord ou du Sud du bassin Maghrébin, suscite un débat depuis plus d'un demi-siècle.

Dans cette note, les grès-quartzites dits numidiens (de plaine sous-marine) sont intégrés dans la séquence de dépôts binaire (fig. 2, 3). Ils ne sont pas aléatoires et apparaissent particulièrement dans les cycles supérieurs (termes $d$, d' et particulièrement $\mathrm{d} 2$ ) de chaque séquence. On peut observer aussi des grès-quartzites dans les discontinuités entre les termes a, b, c, d, e, a', b', c', d' et e'.
Selon les géochimistes (Djarnia, 1990, comm. orale), l'explication est la suivante: les grès-quartzites dits numidiens seraient dus à un milieu acide qui favorise la transformation ou la dissolution des feldspaths en argile, avec enrichissement en quartz $\mathrm{SiO}_{2}$. Cette acidité serait due à une augmentation du gaz carbonique $\mathrm{CO}_{2}$ dans le milieu.

$\mathrm{Au}$ plan stratigraphique, ces grès-quartzites et ces pélites noires pourraient résulter d'un climat chaud qui se manifeste dans le terme $\mathrm{d} 2$ et entre les termes $\mathrm{d}$ et e. Cette limite correspond à la m.f.s. et à la discontinuité $\varphi_{4}$. Dans ces termes la bioturbation est pratiquement absente (diminution de l'oxygène) et le pourcentage des carbonates diminue.

A priori, la tendance est portée vers des dépôts plus proximaux et de plus faible profondeur que les grès-micacés ou arkosiques. De plus, ces pélites noires riches en matière organique seraient aussi des niveaux d'extinction des espèces ou mass extinction dus à une diminution d'oxygène. Ce sont ces extinctions de masse qui seraient à l'origine des
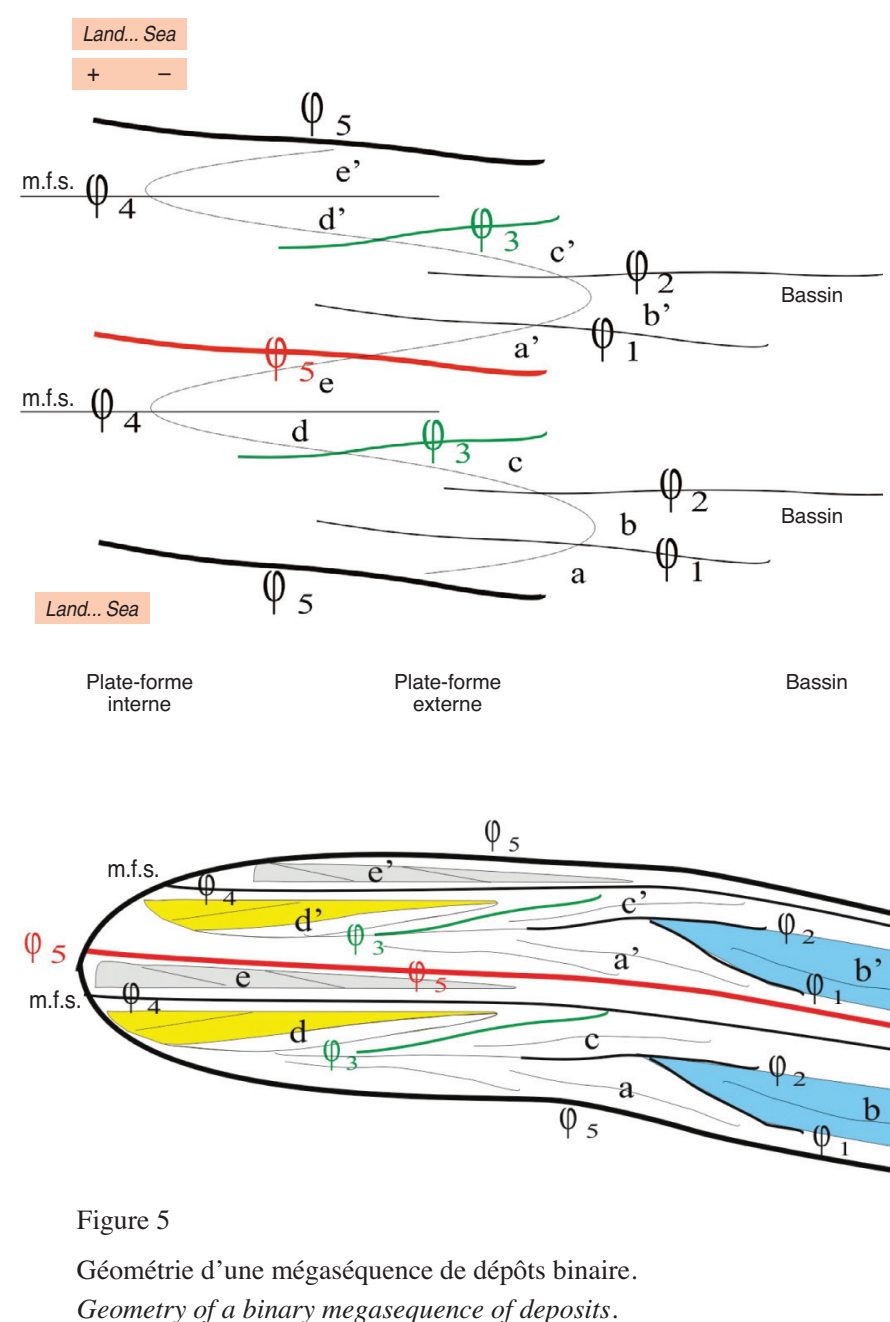
Geometry of a binary megasequence of deposits.
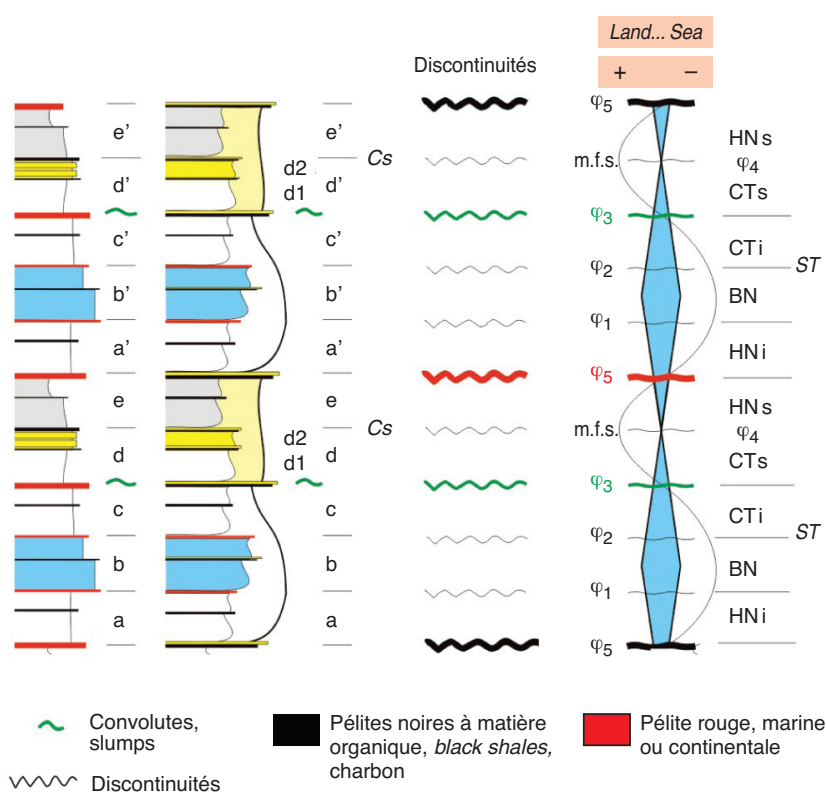

Pélites noires à matière organique, black shales, charbon

Discontinuités

Carbonates, marnes, ou calcilutite

Détritique terrigène
Pélite rouge, marine ou continentale

Gréso-micacé, grès arkosique

Grès-quartzite dit numidien, silex ou roche silicieuse 
roches-mères (blacks shales). En fait, ces faciès grésoquartzitiques seraient donc à l'origine des faciès grésoarkosiques ou gréso-micacés qui ont subi une transformation géochimique.

Il n'est pas nécessaire d'invoquer une source d'apport de la marge sud du bassin Maghrébin pour les faciès numidiens. Ces grès-quartzites proviendraient alors, comme les faciès gréso-micacés, de la marge nord de ce bassin.

\subsection{Les autres séquences et mégaséquences}

D'autres observations ont été faites pour étayer le modèle de séquence de dépôts binaire. D'une part pour vérifier la validité du modèle, et d'autre part pour comprendre les variations de faciès dans d'autres milieux de dépôts.

Nous avons repris certaines séquences dans le bassin Maghrébin, l'Atlas saharien et les tassilis, ainsi que celles d'autres régions décrites dans la littérature.

Que se passe t-il au niveau des phases tectoniques? Pourquoi un climat glaciaire ou la présence de nodules de barytine ou encore d'évaporites? Y a-t-il un chimisme propre aux discontinuités sédimentaires (hiatus) ou tectoniques? Quel est l'intérêt de ces discontinuités dans l'exploration minière ou pétrolière?

\subsubsection{Les variations latérales des séquences de dépôts binaires}

Les variations latérales des séquences de la plaine sousmarine vers la plate-forme, observées sur le terrain ou étudiées dans la littérature, sont comme suit.

Dans le margino-littoral, le charbon remplace la pélite noire. Cette dernière riche en matière organique souligne les discontinuités ou la m.f.s. Cet exemple est pris dans le Carbonifère du Bassin de Béchar, Deleau (1955).

Les silex de la plate-forme remplacent les grès-quartzites dits numidiens (fig. 2, 3) de la plaine sous-marine. Ces alternances ont été observées dans le Cénomanien d'El-Hamel à Bou-Saada (Atlas saharien), à $250 \mathrm{~km}$ au sud-est d'Alger. Ces silex sont stratifiés et alternent avec dix bandes ou termes carbonatés dans une séquence, de trois à quatre mètres d'épaisseur.

Il s'agit d'une séquence de dépôts binaire dont la durée a été estimée à $100 \mathrm{ka}$. Ces alternances seraient dues à des fluctuations climatiques.

Le deuxième cas de figure se rapporte à des silex qui soulignent les discontinuités. Ces silex pourraient correspondre aussi, dans d'autres milieux de dépôts, à des roches siliceuses d'origine chimique ou biochimique.

\subsubsection{La série du Djebel Zima dans le Tell}

Cette série s'étend de l'Albo-aptien à l'Oligocène. Le découpage séquentiel montre un cycle de 31,25 Ma entre le Paléocène et le Bartonien (fig. 4). Ce cycle ou terme a se compose à son tour de cinq termes a, b, c, d et e de 6,25 Ma chacun. Même à cette échelle, le terme $\mathrm{d}$ rapporté au Bartonien présente des faciès terrigènes, comme dans une séquence de $100 \mathrm{ka}$.

Le passage du Bartonien (terme d) au Priabonien (terme e) est marqué par un banc de grès-quartzites pluridécimétrique qui marque la m.f.s. et correspond à la discontinuité $\varphi_{4}$. Ceci pour dire que les faciès de grés-quartzites dits numidiens se développent aussi dans les discontinuités.

On observe une discontinuité entre le Priabonien et l'Oligocène inférieur. Il s'agit de la phase tectonique priabonienne $\varphi_{1}$. Il faut préciser que cette dernière passe inaperçue sur le terrain. C'est grâce au découpage de l'échelle stratigraphique séquentielle que nous l'avons déduite.

\subsubsection{Les séries de Bou-Saada et d'Aflou dans I'Atlas saharien}

Dans l'Atlas saharien, la série sédimentaire de Bou-Saada s'étend du Néocomien au Maestrichtien (fig. 4).

Le découpage séquentiel montre une discontinuité d'ordre 4 entre les carbonates de l'Albien supérieur et les dépôts argilo-évaporitiques du Cénomanien. Il s'agit de la phase tectonique $\varphi_{4}$ (entre les termes d et e) qui correspond à la phase autrichienne et en même temps à une m.f.s. Sur le terrain cette phase n'est pas facilement observable. Elle est matérialisée grâce au découpage séquentiel.

On comprend mieux pourquoi dans les chartes eustatiques, le pic de la transgression marine de l'ère secondaire est situé au Crétacé.

\subsubsection{La série des Tassilis}

La mégaséquence s'étend du Cambrien au Silurien (fig. 4). C'est aussi la série du cycle calédonien de 156,25 Ma.

Le découpage du Cambro-Ordovicien, réalisé par les anciens géologues en unités I, II, III et IV, correspond au découpage en termes a, b, c et d dans cette note.

L'observation la plus importante correspond à la discontinuité d'ordre 3 (en vert) qui matérialise la phase taconique $\varphi_{3}$. Cette dernière délimite les unités III et IV. Cette phase est connue, dans la littérature, comme une phase glaciaire. La glaciation a recouvert le Hoggar et les Tassilis et a permis les dépôts glaciaires de l'unité IV (Ordovicien supérieur). C'était le pôle sud à cette époque.

Dans les séquences plus petites, de l'ordre du $100 \mathrm{ka}$ et $500 \mathrm{ka}$, on trouve plutôt des convolutes ou des slumps au niveau de la discontinuité $\varphi_{3}$.

$\mathrm{Au}$ plan géodynamique, la mégaséquence-type serait constituée de cinq termes a, b, c, d et e. Les trois termes a, b et $c$ ont une tendance franchement marine et les termes $d$ et $e$ ont une tendance continentale à marine.

Une phase tectonique $\varphi_{3}$ se produit entre les termes c et $\mathrm{d}$ pour réduire la tranche d'eau. C'est pour cela que l'on observe des faciès plutôt continentaux (glaciaire pour ce cas) 
dans le terme d1. Le milieu glaciaire présente des vallées incisées au niveau de la discontinuité $\varphi_{3}$.

Au plan séquentiel, le terme d2 correspondrait au B.T.S. ou basal transgressif sandstones de Abbot (1985) en comparant aves les séries sédimentaires du Cambrien des ÉtatsUnis (sandstones Tapeats). Ce sont des niveaux potentiels sur le plan pétrolier.

\section{PERSPECTIVES D'AVENIR}

Il faudra vérifier la validité de ce modèle de séquence de dépôts binaire dans d'autres milieux de dépôts comme le fluviatile, le deltaïque, le lacustre, le glaciaire, la plate-forme carbonatée, etc.

Il reste à préciser sur le terrain la place exacte des minéraux tels que les nodules polymétalliques, la barytine, la glauconie, les phosphates, le fer, les oolites et autres.

A priori et selon des observations partielles, ils devraient se situer au niveau de la m.f.s., de la surface de transgression (entre les termes b et c ou b' et c'), et des dix discontinuités principales $\varphi_{1}, \varphi_{2}, \varphi_{3}, \varphi_{4}, \varphi_{5}$ de la séquence inférieure et $\varphi_{1}$, $\varphi_{2}, \varphi_{3}, \varphi_{4}, \varphi_{5}$ de la séquence supérieure.

Il faudra également préciser la place des évaporites dans les séquences. On les trouve dans les cycles supérieurs (termes d, e et d', e') pour des observations préliminaires. Existent-elles aussi dans les discontinuités?

En stratigraphie séquentielle, il faut étudier davantage la binarité des séquences et la vérifier à différentes échelles pour améliorer le modèle de séquence de dépôts proposé dans cette note.

Pour l'exploration pétrolière, il serait intéressant de se pencher sur les m.f.s., la surface de transgression et les discontinuités. Ces dernières constitueraient des niveaux potentiels en plus du terme $\mathrm{d} 2$.

\section{CONCLUSION}

Une séquence de dépôts binaire a été établie à partir des affleurements de terrain. Elle peut être appliquée à différentes échelles et à différents milieux de dépôts.

C'est une séquence de dépôts composée de deux séquences (inférieure et supérieure) de même durée. Chacune des séquences comprend cinq termes a, b, c, d, e et a', b', c', d', e' de durées identiques. Ces dix termes sont délimités entre eux par dix discontinuités: $\varphi_{1}, \varphi_{2}, \varphi_{3}, \varphi_{4}, \varphi_{5}$ de la séquence inférieure et $\varphi_{1}, \varphi_{2}, \varphi_{3}, \varphi_{4}, \varphi_{5}$ de la séquence supérieure.

Ces deux séquences se subdivisent à leur tour en deux cycles chacune. Les cycles inférieurs des deux séquences représentent $3 / 5^{\mathrm{e}}$ en durée et les cycles supérieurs $2 / 5^{\mathrm{e}}$. Au total, il y a quatre cycles et quatre discontinuités importantes.

On distingue deux m.f.s. qui se situent au centre des cycles supérieurs. La section condensée ou condensed section Cs se situe au niveau de la m.f.s. ou maximum flooding surface et de la discontinuité $\varphi_{4}$.

Ce modèle de séquence de dépôts binaire sera amélioré dans le futur avec de nouvelles observations sur le terrain.

Huit ordres de séquences de dépôts binaires et emboîtées ont été déterminés. Ce sont du premier au huitième ordre des sous-multiples de cinq. Les différents ordres de séquences de dépôts binaires et la hiérarchie des différents ordres de discontinuités ont permis d'élaborer une échelle stratigraphique séquentielle. Cette dernière sera corrigée au fur et à mesure de nouvelles données de terrain.

$\mathrm{Au}$ plan géodynamique, la mégaséquence-type serait constituée de cinq termes a, b, c, d et e. Les trois termes a, b et $\mathrm{c}$ ont une tendance franchement marine et les termes d et e ont une tendance continentale à marine.

Une phase tectonique $\varphi_{3}$ se produit entre les termes $\mathrm{c}$ et $\mathrm{d}$ pour réduire la tranche d'eau. C'est pour cela que l'on observe des faciès plutôt continentaux (fluviatile, éolien, lacustre, glaciaire, évaporitique, etc.) dans le terme d.

Les milieux fluviatile et glaciaire peuvent présenter des vallées incisées au niveau de la discontinuité $\varphi_{3}$.

Au plan minier, c'est dans les m.f.s. et les discontinuités principales que semblent se développer les minerais sédimentaires.

Au plan pétrolier, les deux cycles supérieurs des mégaséquences de dépôts binaire seraient le siège probable des roches-mères et des roches-réservoirs favorables à la genèse et à l'accumulation des hydrocarbures.

Les termes d et d', qui présentent des vallées incisées, et particulièrement le sous-terme d2, qui correspond à la m.f.s. ou au Basal Transgressif Sandstones (BTS de Abbot, 1985), seraient des niveaux intéressants dans l'exploration.

Le troisième objectif serait l'exploration dans une mégaséquence de la surface de transgression (entre les termes b et $\mathrm{c}$ ou b' et c'), des limites entre les termes a, b, c, d, e, a', b', c', d' et e', ou des dix discontinuités.

\section{REMERCIEMENTS}

Je remercie Messieurs Michel Hoffert et Max Deynoux de l'Université Louis Pasteur, ainsi que Monsieur Christian Ravenne de l'Institut français du pétrole, pour m'avoir soutenu et encouragé dans mes travaux.

Je remercie également Monsieur M.R. Djarnia, géochimiste au Centre de Recherches et Développement (C.R.D.) de Sonatrach à Boumerdès, pour avoir analysé et interprété les échantillons de pélites noires.

\section{RÉFÉRENCES}

Abbot W.O. (1985) The recognition and mapping of a basal transgressive sand from outcrop, subsurface, and seismic data, Berg, Wool Verton D.-G. (eds.), Seismic stratigraphy II - an integrated approach, Amer. Ass. Petrol. Geol., Mem. 39, 157-167. 
Bouma A.H. (1962) Sedimentology of some flysch deposits. A graphic approach to facies interpretation, Elsevier Publ. Co., Amsterdam, $168 \mathrm{p}$.

Cross T.A. (1988) Controls on coal distribution in transgressive regressive cycles, Upper Cretaceous, Western Interior, USA, in Sea level change an integraded approach, Hasting B., Kendall C. (eds.), Soc. Econ. Paleont. Miner. Spec. Publ., pp. 371-380.

De Boer P.L., Wonders A.A.H. (1984) Astronomically induced rhythmic bedding in Cretaceous pelagic sediments near Moria (Italy), in Milankovitch and climate, Berger A. et al. (eds.), Published by Reidel D., Publishing Company, Holland, pp. 17-190.

Deleau P. (1955) La sédimentation du faisceau houiller $d u$ Namurien du Sud-Oranais. Les érosions subcontemporaines $d u$ dépôt, Public. du Service de la Carte Géol. de l'Algérie (Nouvelle série); Bull. n 5, Trav. des Collab. 1954, 14 fig. d. t., 4 h.-t., pp. 311-353.

Gilbert G.K. (1895) Sedimentary measurement of Cretaceous time, J. Geol. 3-2, 121-127.
Goodwin P.W., Anderson E.J. (1985) Ponctuated aggradational cycles: a general hypothesis of episodic stratigraphic accumulation, Univ. of Chicago, J. Geol. 93, 515-533.

Rohde M., Muller R. (2005) Life comes and goes unexplained cycles. Fossil record yields surprising finding, The Berkeley lab VIEW, UC Berkeley, $14 \mathrm{p}$.

Sloss L.L. (1963) Sequences in the cratonic interior of North America, Geol. Soc. Am. Bull. 74, 93-114.

Sloss L.L. (1988) Tectonic evolution of the craton in Phanerozoic time, in Sloss L.L. (ed.), Sedimentary Cover - North American Craton, U.S.: Geologic Society of America, Decade of North American Geology, v. D-2, pp. 25-51.

Vail P.R., Mitchum R.H., Todd R.G., Widmier J.M., Thompson S., Sangree J.B., Bubb J.N., Hatlelid W.G. (1977) Seismic stratigraphy and global changes of sea level, Payton C.E. (ed.), Amer. Ass. Petrol. Geol. Mem. 26, 49-212.

Manuscrit final reçu en avril 2009 Publié en ligne en septembre 2009 